\title{
A Multi-window Fractional Evolutionary Spectral Analysis
}

\author{
YALÇIN ÇEKİÇ, LÜTFİ İHSAN YAZICI and AYDIN AKAN ${ }^{1}$ \\ Istanbul University, Department of Electrical and Electronics Engineering \\ Avcilar, Istanbul, 34580, TURKEY
}

\begin{abstract}
In this work, we present a multiple window Evolutionary Spectral analysis on a nonrectangular time-frequency lattice based on a discrete fractional Gabor expansion. The traditional Gabor expansion uses a fixed, and rectangular time-frequency plane tiling. Many of the practical signals such as speech, music, etc., require a more flexible, non-rectangular time-frequency lattice for a compact representation. The proposed method uses a set of basis functions that are related to the fractional Fourier basis and generate a parallelogram-shaped tiling. Simulation results are given to illustrate the performance of our algorithm.
\end{abstract}

Key-Words: - Time-frequency analysis, Gabor expansion, Fractional Fourier Transform, Evolutionary Spectral analysis.

\section{Introduction}

Time-frequency (TF) analysis provides a characterization of signals in terms of joint time and frequency content $[1,2]$. Evolutionary Spectrum (ES) is one of the TF analysis methods [3] which is based on the decomposition of signals into sinusoids with random and time-varying amplitudes. In our previous work, we present a method to estimate the ES of discrete-time, non-stationary signals using Gabor expansions [4]. The Gabor expansion is a TF decomposition which represents a signal in terms of time and frequency translated basis functions [5, 6]. Gabor basis functions are obtained by shifting and modulating with a sinusoid a single window function, which results in a fixed and rectangular TF plane tiling. However, many of the practical signals such as speech, music, biological, and seismic signals have timevarying frequency nature that is not appropriate for this type of analysis [7, 8, 9]. Thus the Gabor expansion of such signals will require large number of coefficients yielding a poor TF localization $[10,11]$.

\footnotetext{
1 This work was supported by the Research Fund of The University of Istanbul, Project numbers: UDP$145 / 23052003$ and $107 / 15052003$.
}

Therefore, the ES estimate we obtain by using the Gabor expansion suffers from a TF resolution problem. Here we present an ES analysis method based on a new, fractional Gabor expansion that uses a more flexible, parallelogram-shaped TF lattice [12]. The basis functions of the proposed expansion are related to the fractional Fourier series basis [13].

\section{Evolutionary Spectral Analysis}

In [4], we present an Evolutionary Spectral (ES) estimate based on a multi-window Gabor expansion. In the following, we briefly present the discrete multi-window Gabor expansion, and our ES estimation.

\subsection{A Multi-window Gabor Expansion}

Multi-window Gabor expansion [4] represents a signal in terms of scaled and time and frequency shifted basis functions and is given for a finitesupport signal $x(n), 0 \leq n \leq N-1$ by

$$
x(n)=\frac{1}{I} \sum_{i=0}^{I-1} \sum_{m=0}^{M-1} \sum_{k=0}^{K-1} a_{i, m, k} \tilde{g}_{i, m, k}(n)
$$

where the basis functions are

$$
\tilde{g}_{i, m, k}(n)=\tilde{g}_{i}(n-m L) e^{j \omega_{k} n}
$$


and $\omega_{k}=2 \pi k L^{\prime} / N$. Here the synthesis window $\tilde{g}_{i}(n)$ is a periodic version by $N,\left(\tilde{g}_{i}(n)=g_{i}(n+\right.$ $r N), r$ integer) of a time-scaled Gabor window as

$$
g_{i}(n)=2^{i / 2} g\left(2^{i} n\right), i=0,1, \ldots, I-1 .
$$

and $\left.g_{(} n\right)$ which is normalized to unit energy for definiteness [6]. $I$ is the number of windows or resolution levels used and Gabor expansion parameters $M, K, L$, and $L^{\prime}$ are positive integers constrained by $M L=K L^{\prime}=N$ where $M$ and $K$ are the number of analysis samples in time and frequency, respectively. The Gabor coefficients can be calculated using an auxiliary function $\gamma_{i}(n)$ called the biorthogonal window or dual function of $g_{i}(n)$, i.e.,

$$
a_{i, m, k}=\sum_{n=0}^{N-1} x(n) \tilde{\gamma}_{i, m, k}^{*}(n)
$$

where $\tilde{\gamma}_{i, m, k}(n)=\tilde{\gamma}_{i}(n-m L) e^{j \frac{2 \pi k}{K} n}$ and $\tilde{\gamma}_{i}(n)$ analysis window is solved from the biorthogonality condition between $\tilde{g}_{i}(n)$ and $\tilde{\gamma}_{i}(n)[6]$ :

$$
\sum_{n=0}^{N-1} \tilde{h}_{i}(n+m K) e^{-j \frac{2 \pi}{L} k n} \tilde{\gamma}_{i}^{*}(n)=\frac{L}{K} \delta_{m} \delta_{k}
$$

for $0 \leq m \leq L^{\prime}-1, \quad 0 \leq k \leq L-1$. Gabor analysis basis $\left\{\tilde{\gamma}_{i, m, k}(n)\right\}$ with fixed window and sinusoidal modulation tiles the TF plane in a rectangular fashion. An example of such a sampling geometry is shown in Fig. 1. Such methods usually provide signal representations with poor TF resolution [7].

\subsection{Evolutionary Spectral Analysis}

We obtain in [4] an evolutionary spectral estimate based on the Gabor coefficients. We consider the following discrete-time, discrete-frequency model for finite-extent, deterministic signals that is analogous to the Wold-Cramer representation of nonstationary random signals:

$$
x(n)=\sum_{k=0}^{K-1} A\left(n, \omega_{k}\right) e^{j \omega_{k} n}, \quad 0 \leq n \leq N-1,
$$

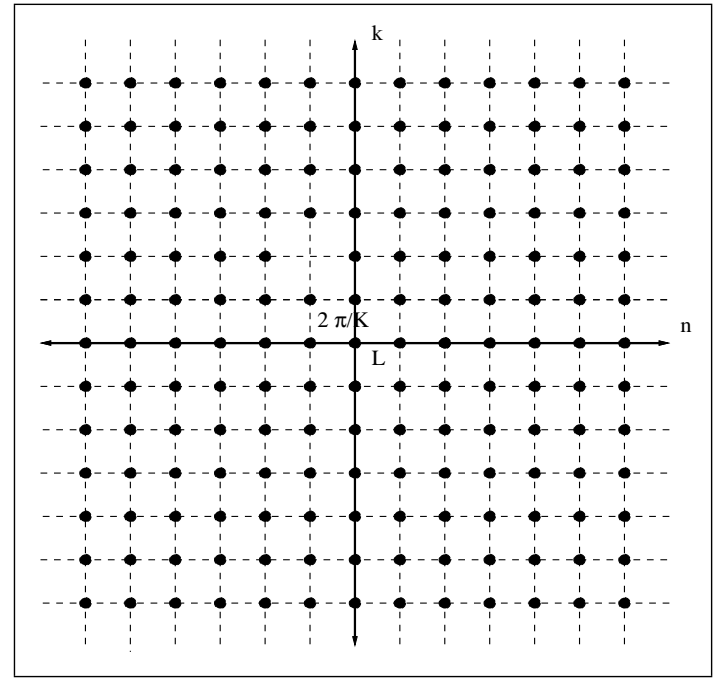

Fig. 1. Rectangular time-frequency plane tiling used in the above Gabor expansion.

where $\omega_{k}=2 \pi k / K$, and $A\left(n, \omega_{k}\right)$ is a TF kernel. Comparing the two representations of $x(n)$ in (1) and (5), we have that the kernel is

$$
\begin{aligned}
A\left(n, \omega_{k}\right) & =\frac{1}{I} \sum_{i=0}^{I-1} \sum_{m=0}^{M-1} a_{i, m, k} \tilde{g}_{i}(n-m L) \\
& =\sum_{\ell=0}^{N-1} x(\ell) \mathrm{w}(n, \ell) e^{-j \omega_{k} \ell}
\end{aligned}
$$

where we substituted for the coefficients $\left\{a_{i, m, k}\right\}$ and defined the time-varying window

$$
\mathrm{w}(n, \ell)=\frac{1}{I} \sum_{i=0}^{I-1} \sum_{m=0}^{M-1} \tilde{\gamma}_{i}^{*}(\ell-m L) \tilde{g}_{i}(n-m L) .
$$

Then the evolutionary spectrum of $x(n)$ is obtained by

$$
S_{E S}\left(n, \omega_{k}\right)=\frac{1}{K}\left|A\left(n, \omega_{k}\right)\right|^{2},
$$

where the factor $1 / K$ is used for proper energy normalization. The above ES analysis method with a fixed, and rectangular TF lattice yields a poor resolution. Several approaches have been proposed to improve the resolution of such sinusoidal representations: some of them are averaging estimates obtained using different windows [4], and maximizing energy concentration measures [7, 9, 14]. In recent works, representations on a non rectangular TF grid has attracted a considerable attention $[8,9,12]$. A non rectangular lattice is 
more appropriate for the TF analysis of signals with time-varying frequency content. This is the motivation of our fractional evolutionary spectral analysis.

\section{Fractional Gabor Expansion}

We define a discrete fractional multi-window Gabor expansion for $x(n), 0 \leq n \leq N-1$, as follows:

$$
x(n)=\frac{1}{I} \sum_{i=0}^{I-1} \sum_{m=0}^{M-1} \sum_{k=0}^{K-1} a_{i, m, k, \alpha} \tilde{g}_{i, m, k, \alpha}(n)
$$

where $a_{i, m, k, \alpha}$ are the fractional Gabor coefficients, $\alpha$ is the order of the fraction, and the basis functions are

$$
\tilde{g}_{i, m, k, \alpha}(n)=\tilde{g}_{i}(n-m L) W_{\alpha, k}(n)
$$

Here $\tilde{g}_{i}(n)$ is defined as above, and $W_{\alpha, k}(n)$ is the fractional kernel,

$$
W_{\alpha, k}(n)=e^{j\left[-\frac{1}{2}\left(n^{2}+\left(\omega_{k} \sin \alpha\right)^{2}\right) \cot \alpha+\omega_{k} n\right]}
$$

which is similar to the Fractional Fourier Series basis functions presented in [13], and again $\omega_{k}=$ $2 \pi k / K$. Above basis functions with this fractional kernel generate a parallelogram-shaped TF sampling geometry given in Fig. 2. The expansion in (9) reduces to the above sinusoidal Gabor expansion for $\alpha=\pi / 2$. The parameters $M, K$, $L$, and $L^{\prime}$, are same as in the traditional Gabor expansion. For numerically stable solutions we need that $L \leq K$. The case where $L=K$ is called the critical sampling, and the case where $L<K$ is the oversampling. In our derivations, we always consider the general, oversampled case. The Gabor coefficients can be evaluated as before by

$$
a_{i, m, k, \alpha}=\sum_{n=0}^{N-1} x(n) \tilde{\gamma}_{i, m, k, \alpha}^{*}(n)
$$

where the analysis functions are

$$
\tilde{\gamma}_{i, m, k, \alpha}(n)=\tilde{\gamma}_{i}(n-m L) W_{\alpha, k}(n)
$$

and $\tilde{\gamma}_{i}(n)$ is periodic version of a $\gamma_{i}(n)$ that is solved from a fractional biorthogonality condition between $g_{i}(n)$ and $\gamma_{i}(n)$.

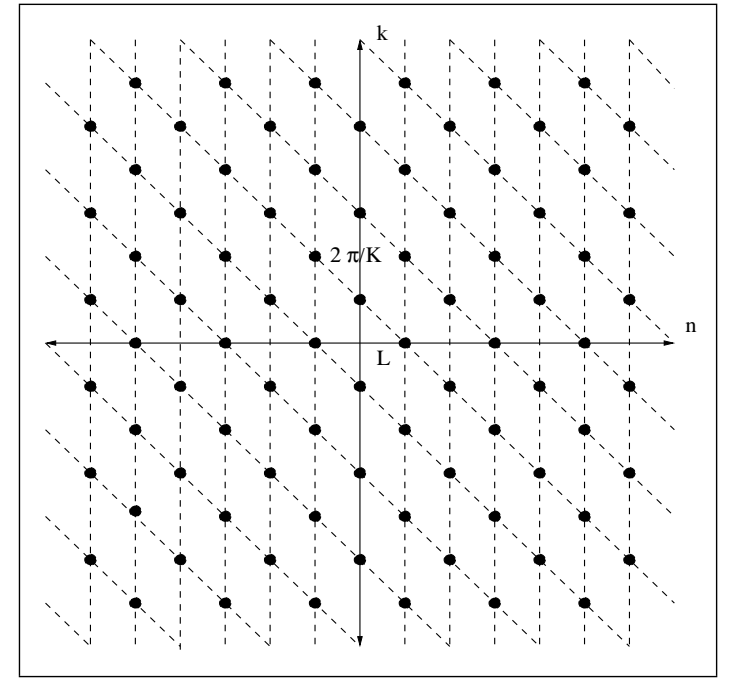

Fig. 2. Time-frequency plane lattice generated by the fractional Gabor expansion.

To obtain the completeness condition for the fractional Gabor basis for scale $i$, substitute (10) in (9):

$$
\begin{aligned}
x(n) & =\sum_{m=0}^{M-1} \sum_{k=0}^{K-1}\left(\sum_{\ell=0}^{N-1} x(\ell) \tilde{\gamma}_{i}^{*}(\ell-m L) W_{\alpha, k}^{*}(\ell)\right) \\
& \times \tilde{g}_{i}(n-m L) W_{\alpha, k}(n) \\
& =\sum_{\ell=0}^{N-1} \sum_{m=0}^{M-1} \sum_{k=0}^{K-1} \tilde{g}_{i}(n-m L) \tilde{\gamma}_{i}^{*}(\ell-m L) \\
& \times e^{j\left[-\frac{1}{2}\left(n^{2}-\ell^{2}\right) \cot \alpha+\omega_{k}(n-\ell)\right]}
\end{aligned}
$$

From the above equation, we obtain the completeness relation for basis $\left\{\tilde{g}_{i, m, k, \alpha}(n)\right\}$ as

$$
\begin{gathered}
\sum_{m=0}^{M-1} \sum_{k=0}^{K-1} \tilde{g}_{i}(n-m L) \tilde{\gamma}_{i}^{*}(\ell-m L) e^{j\left[-\frac{1}{2}\left(n^{2}-l^{2}\right) \cot \alpha\right]} \\
\times e^{j \omega_{k}(n-\ell)}=\delta(n-\ell)
\end{gathered}
$$

The fractional biorthogonality condition that we need to solve the analysis or dual function $\gamma_{i}(n)$ is obtained from the above completeness relation using a discrete Poisson sum formula as:

$$
\begin{array}{r}
\sum_{n=0}^{N-1} \tilde{g}_{i}^{*}(n+m K) e^{j k \frac{2 \pi}{L}(n+m K)} \tilde{\gamma}_{i}(n) \\
\quad \times e^{j\left(n m K+\frac{m^{2} K^{2}}{2}\right) \cot \alpha}=\frac{L}{K} \delta_{m} \delta_{k} \\
0 \leq m \leq L^{\prime}-1, \quad 0 \leq k \leq L-1
\end{array}
$$




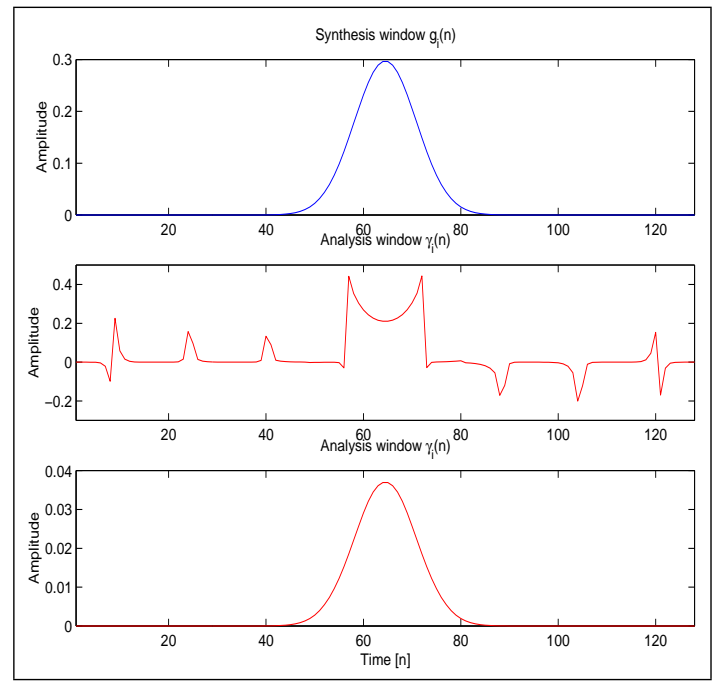

Fig. 3. A Gauss synthesis window (top figure), and its biorthogonal windows in critical (middle) and oversampling (bottom) cases.

Completeness and biorthogonality conditions given in equations (11) and (11) reduce to the conditions in the traditional case [6] for $\alpha=\pi / 2$. This indicates that the above fractional expansion is the generalization of the Gabor expansion. In Fig. 3, we show a Gauss window $g_{i}(n), n=0,1, \cdots, 127$ on the top figure, and its biorthogonal $\gamma_{i}(n)$ for two different set of sampling parameters obtained by solving equation (11) for $\alpha=\pi / 4$. The window in the middle is obtained using $L=16, K=$ 16 that is the critical sampling. The window at the bottom is calculated with $L=8, K=64$ as an example of the oversampling.

\section{Fractional Evolutionary Spectrum}

In this section we present a fractional evolutionary spectral analysis method based on the above Gabor expansion. Here we consider the discretetime, and discrete-frequency representation for $x(n)$ given in equation (1). Comparing this with the fractional Gabor representation in (9), we get the time-frequency kernel using window $\tilde{g}_{i}(n)$ as

$$
\begin{aligned}
A\left(n, \omega_{k}\right) & =\frac{1}{I} \sum_{i=0}^{I-1} \sum_{m=0}^{M-1} a_{i, m, k, \alpha} \tilde{g}_{i}(n-m L) \\
& \times e^{-j \frac{1}{2}\left(n^{2}+\left(\omega_{k} \sin \alpha\right)^{2}\right) \cot \alpha}
\end{aligned}
$$

After replacing for the coefficients in (10) we have that

$$
\begin{aligned}
A\left(n, \omega_{k}\right) & =\sum_{\ell=0}^{N-1} x(\ell) \frac{1}{I} \sum_{i=0}^{I-1} \mathrm{p}_{i}(n, \ell) e^{-j \omega_{k} \ell} \\
& =\frac{1}{I} \sum_{i=0}^{I-1} A_{i}\left(n, \omega_{k}\right)
\end{aligned}
$$

where we defined the time-varying, fractional- modulated window,

$$
\begin{aligned}
\mathrm{p}_{i}(n, \ell) & =\sum_{m=0}^{M-1} \tilde{g}_{i}(n-m L) \tilde{\gamma}_{i}^{*}(\ell-m L) \\
& \times e^{\left.j \frac{1}{2}\left(\ell^{2}-n^{2}\right)\right) \cot \alpha}
\end{aligned}
$$

The equation in (13) can be interpreted as the average of short-time Fourier transforms with scaled, time-dependent and non-sinusoidal modulated windows, $p_{i}(n, \ell)$. The fractional evolutionary spectrum is then obtained as before. $\mathrm{p}_{i}(n, \ell)$ can be calculated independent of the signal and then the calculation of the ES can be achieved very efficiently using FFT. It can also be shown that this ES estimate can preserve the energy of the signal if the windows $p_{i}(n, \ell)$ satisfy the condition:

$\sum_{n}\left|p_{i}(n, \ell)\right|^{2}=1, \quad n, \ell \in[0, N-1], \forall i$

In Fig. 4, we show an example of this time-varying fractional window $p_{i}(n, \ell)$ for $\alpha=\pi / 4$ at time instants $n=32$ and $n=80$ and their spectrogram together.

\section{Experimental Results}

We consider a signal composed of two chirps, with $\pi / 4$ and $3 \pi / 8$ rad. angles. Using our fractional method, we analyzed the signal with two different fractional orders. Fig. 5 shows the ES estimate of this two-chirp signal after compensating for the rotation caused by the fractional analysis with $\alpha=\pi / 4$, and sampling parameters $L=$ $4, K=128$, and $I=4$. We also show the ES estimate using $\alpha=3 \pi / 8$ in Fig. 6 . Notice that in both figures, the component that is matched by the analysis angle is represented with higher concentration. To compare the results of our proposed fractional method, we applied the sinusoidal multiwindow evolutionary spectrum explained in section 2, using the same Gabor parameters.As shown, 


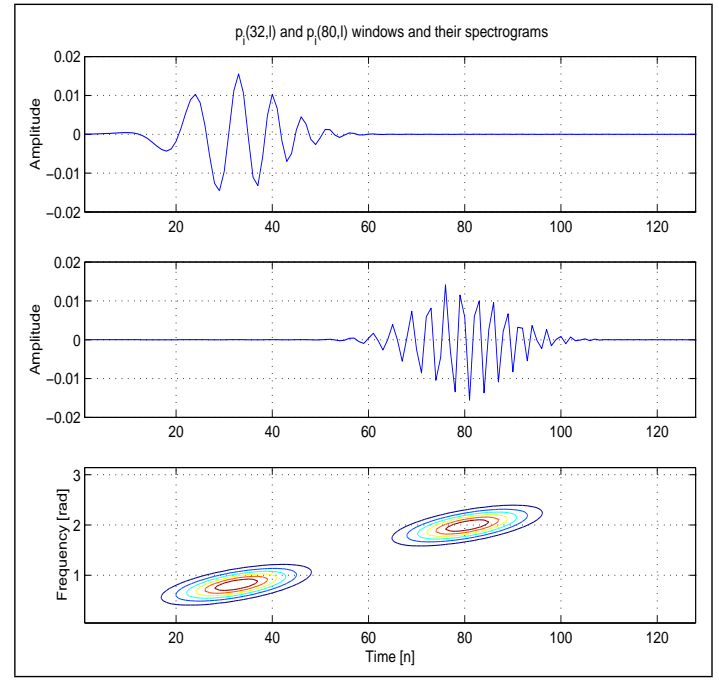

Fig. 4. The time-varying chirp window used in ES for $n=32$ (top figure), $n=80$ (middle) and their spectrogram (bottom).

sinusoidal ES given in Fig. 7 results poor TF localization for both components.

\section{Conclusions}

In this paper, we present a method for fractional evolutionary spectral analysis of discrete-time, nonstationary signals. The evolutionary kernel is obtained via the coefficients of a fractional Gabor expansion. We give the completeness and biorthogonality conditions of this new expansion. Simulations show that the fractional method gives high resolution ES results if the analysis fraction order match the frequency content of the signal. Hence, for an arbitrary signal, the fraction order $\alpha$ can be chosen from a set of values $\left\{\alpha_{1}, \alpha_{2}, \cdots, \alpha_{p}\right\}$ by maximizing a concentration criteria similar to the method used in [7, 14].

\section{References:}

[1] Hlawatsch F. and Boudreaux-Bartels, G. F. "Linear and quadratic time-frequency signal representations," IEEE Signal Processing Magazine, vol. 9, No. 2, pp. 21-67, Apr. 1992.

[2] Cohen, L., Time-Frequency Analysis. Prentice Hall, Englewood Cliffs, NJ, 1995.
[3] Priestley, M.B., "Evolutionary Spectra and Non-stationary Processes," J. of Royal Statistical Society, B, Vol.27, No. 2, pp. 204237, 1965.

[4] Akan, A., and Chaparro, L.F., "Multiwindow Gabor Expansion for Evolutionary Spectral Analysis," Signal Processing, Vol. 63, pp. 249-262, Dec. 1997.

[5] Gabor, D., "Theory of Communication," J. IEE, Vol. 93, pp. 429-459, 1946.

[6] Wexler, J., and Raz, S., "Discrete Gabor Expansions," Signal Processing, Vol. 21, No. 3, pp. 207-220, Nov. 1990.

[7] Jones, D.L., and Parks, T.W., "A High Resolution Data-Adaptive Time-Frequency Representation," IEEE Trans. on Signal Proc., Vol. 38, No. 12, pp. 2127-2135, Dec. 1990.

[8] Mallat, S., and, Zhang, Z., "Matching Pursuit with Time-Frequency Dictionaries," IEEE Trans. on Signal Proc., Vol. 41, No. 12, pp. 3397-3415, Dec. 1993.

[9] Baraniuk, R.G., and Jones, D.L., "Shear Madness: New Orthonormal Bases and Frames Using Chirp Functions," IEEE Trans. on Signal Proc., Vol. 41, No. 12, pp. 3543-3549, Dec. 1993.

[10] Jones, D.L., and Baraniuk, R.G., "A Simple Scheme For Adapting Time-Frequency Representations," IEEE Trans. on Signal Proc., Vol. 42, No. 12, pp. 3530-3535, Dec. 1994.

[11] Bultan A., "A Four-Parameter Atomic Decomposition of Chirplets," IEEE Tans. on Signal Proc., Vol. 47 pp. 731-745, 1999.

[12] Bastiaans, M.J., and van Leest, A.J., "From the Rectangular to the Quincunx Gabor Lattice via Fractional Fourier Trasformation," IEEE Signal Proc. Letters, Vol. 5, No. 8, pp. 203-205, 1998. 
[13] Pei, S.C., Yeh, M.H., and Luo, T.L., "Fractional Fourier Series Expansion for Finite Signals and Dual Extension to DiscreteTime Fractional Fourier Transform," IEEE Trans. on Signal Proc., Vol. 47, No. 10, pp. 2883-2888, Oct. 1999.

[14] Akan, A., and Chaparro, L.F., "Evolutionary Chirp Representation of Non-stationary Signals via Gabor Transform,", Signal Processing, Vol. 81, No. 11, pp. 2429-2436, Nov. 2001.

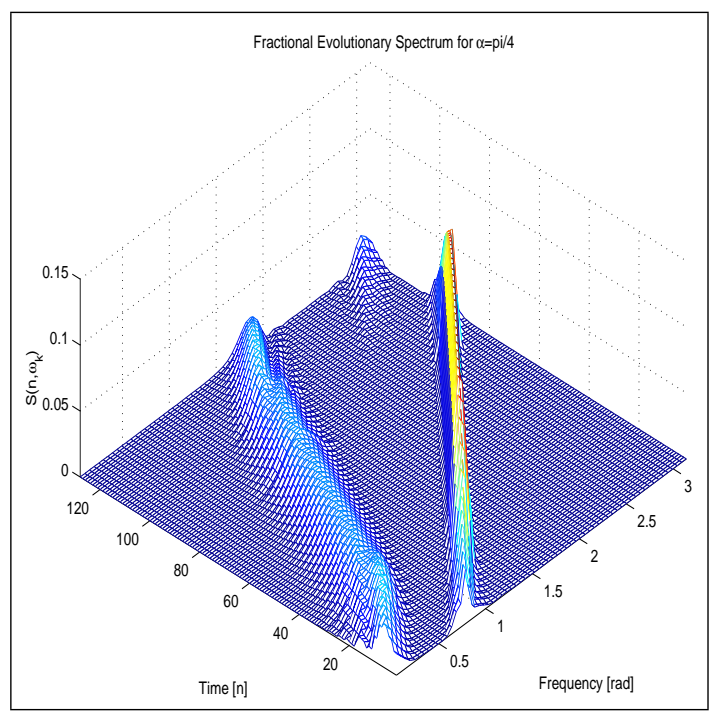

Fig. 5. Fractional ES estimate using $\alpha=\pi / 4$.

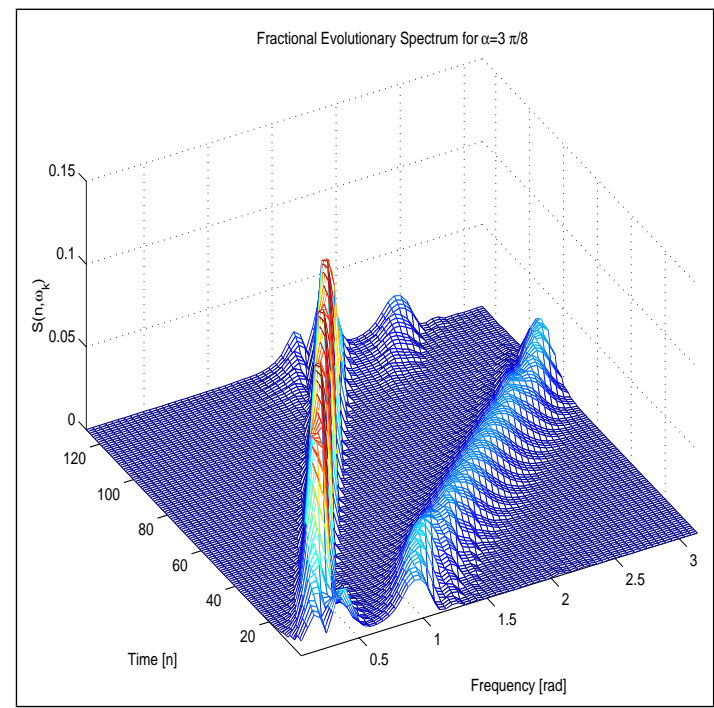

Fig. 6. ES estimate using $\alpha=3 \pi / 8$.

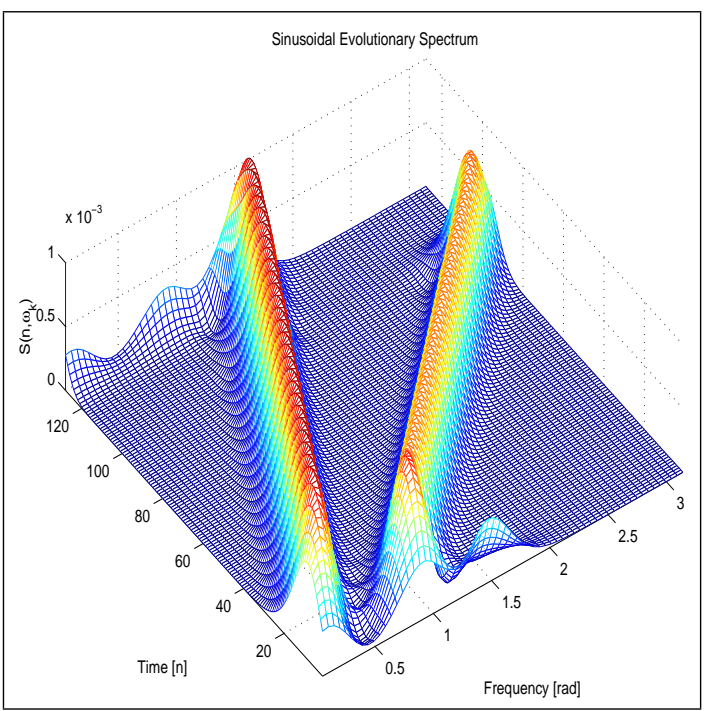

Fig. 7. Multi-window ES estimate of the twochirp signal. 